\title{
A CRIANÇA HOSPITALIZADA: \\ ANÁLISE DE UM PROGRAMA DE ATIVIDADES \\ PREPARATÓRIAS PARA O PROCEDIMENTO \\ MÉDICO DE INALAÇÃO*
}

\author{
THE HOSPITALIZED CHILD: AN ANALYSIS OF \\ A PSYCHOLOGICAL INTERVENTION PROGRAM ON THE \\ PREPARATION FOR AN INHALATION MEDICAL PROCEDURE
}

Maria Rita Zoéga SOARES ${ }^{1}$

Edda BOMTEMPO²

\begin{abstract}
RESUMO
A hospitalização infantil beneficia-se com intervenção psicológica para promover a modificação do comportamento de pacientes. Foram propostos procedimentos observacionais e experimentais para avaliar um programa de atendimento a crianças expostas ao procedimento médico de inalação, tendo como objetivo a diminuição da freqüência de ocorrência de comportamentos concorrentes e o aumento nos comportamentos de adesão. A Observational Scale of Distress Behavior (OSDB) possibilitou o registro das categorias comportamentais. Participaram do estudo, 20 crianças, distribuídas em grupos experimental e controle. Crianças pertencentes ao grupo experimental submeteram-se a um programa que incluiu estratégias relacionadas à leitura, à simulação, ao relaxamento e à fantasia. $A$ análise dos dados demonstrou que os participantes do grupo experimental apresentaram um padrão comportamental mais adaptativo. Relações funcionais foram explicitadas para melhor compreensão do comportamento dos participantes. Os resultados obtidos pretendem subsidiar o desenvolvimento de programas de preparação para procedimentos médicos.

Palavras-chave: criança, hospital, psicologia pediátrica, procedimentos médicos, intervenção psicológica.

(*) O presente texto é parte da Tese de Doutorado apresentada junto ao Instituto de Psicologia da Universidade de São Paulo

(1) Doutora em Psicologia da Universidade Estadual de Londrina. Endereço para correspondência: Universidade Estadual de Londrina - Campus Universitário - Londrina/PR Cep: 86051-900. E-mail: mrzoega@sercomtel.com.br

(2) Doutora em Psicologia - Livre-Docente junto ao Instituto de Psicologia da Universidade de São Paulo, orientadora da Tese
\end{abstract} em novembro/2002. A autora foi bolsista da CAPES. de Doutorado da autora. 


\begin{abstract}
Children's hospitalization needs psychological intervention to promote patient's better behavior. Experimental and observational procedures were proposed to assess an assistantship program for hospitalized children during an inhalation medical procedure, observing the decrease of concurrent behavior and the increase of adequate behavior. The Observational Scale of Distress Behavior (OSDB) was used to register the infant behavior categories. This research was carried out with 20 children distributed in an experimental group and a control group. Children from the experimental group were submitted to program which included the development of reading related strategies, simulations, relaxation techniques and fantasy. The data analysis demonstrated that the experimental group showed a more adequate behavior pattern. Furthermore, the functional relationships in the hospital environment were better understood thus improving patient behavior. Results from this study may subsidize the development of psychological programs for medical procedures in hospitals.
\end{abstract}

Key words: child, hospital, pediatrics psychology, medical procedures, psychological intervention.

\section{INTRODUÇÃO}

Durante seu desenvolvimento, a criança explora e interage com seu meio de forma contínua e recíproca. Por meio desta interação mútua, à medida que características ambientais sejam favoráveis e oportunidades sejam oferecidas, ocorrem modificações em seu repertório comportamental e na natureza funcional do meio (Guimarães, 1988).

Assim, a implementação de serviços de atendimento no hospital deve considerar um planejamento ambiental da instituição para que possam ser efetuadas orientações em relação ao desenvolvimento comportamental da criança. Há necessidade de compreensão da relação funcional entre o paciente e o ambiente em que são dispensados os cuidados com o tratamento (Costa Jr., 1999).

Zannon (1991) considerou relevante o incremento de pesquisas que enfoquem o desenvolvimento de repertórios comportamentais e a relação funcional entre organismo e ambiente. Para a autora respostas a pessoas e lugares estranhos estão relacionadas, não somente à circunstância em si ou a um processo de ordem maturacional, mas também ao arranjo de oportunidades combinadas entre aspectos físicos e sociais, entre estímulos desconhecidos e familiares.

A reação da criança diante de eventos excitantes, irritantes, felizes e amedrontadores exige adaptação, visto que inclui mudanças psicológicas, físicas e químicas no seu organismo. Alguns eventos são fatores que têm um potencial para causar desequilíbrio entre demandas ambientais e recursos individuais e têm sido relacionados a comportamentos-problema, particularmente quando esses eventos alteram, substancialmente, o ambiente da criança (Lazarus \& Folkman, 1984; Rushforth, 1999).

Atualmente, considera-se que a experiência de enfrentar a doença e a hospitalização pode constituir uma oportunidade para que a criança adquira determinados padrões comportamentais mais adaptativos. A hospitalização pode representar uma oportunidade para que o paciente aprenda mais sobre a doença e o funcionamento de seu corpo; descubra sobre as profissões da área da saúde; adquira habilidades de enfrentamento; demonstre capacidade para tomar decisões, independência, autocontrole e autocon- 
fiança, tornando-se participante mais ativo em decisões clínicas. Tal perspectiva é congruente com o crescente e atual reconhecimento dos direitos da criança sobre a informação de sua condição (Rushforth, 1999).

Estudos sugerem que a sensibilidade comportamental da criança a arranjos ambientais específicos aumenta a probabilidade da alteração do comportamento, permitindo o estabelecimento de repertórios comportamentais diferenciados. Tais repertórios podem ser representados pelo estabelecimento de respostas de adesão ao tratamento, comportamentos colaborativos ou participação ativa em processos de tomada de decisão (Adams-Greenly, 1991; Bearison \& Mulhern, 1994; Carpenter, 1991; Zannon, 1991).

Um analista do comportamento tem como tarefa identificar contingências que estão operando (ou inferir quais as que podem ou devem ter operado), quando se depara com determinados comportamentos ou processos comportamentais em andamento, bem como propor, criar ou estabelecer relações de contingência para o desenvolvimento de certos processos comportamentais. É por meio da manipulação de contingências que se podem estabelecer ou instalar comportamentos, alterar padrões, assim como reduzir, enfraquecer ou eliminar comportamentos dos repertórios dos organismos (Souza, 1997).

A presente pesquisa teve como objetivo avaliar os efeitos de um Programa de Atividades (que incluiu a leitura, a simulação, o relaxamento e a fantasia) sobre o repertório comportamental de crianças, no sentido de facilitar sua adaptação ao procedimento médico de inalação. Os objetivos específicos prevêem a diminuição da freqüência de emissão de comportamentos concorrentes durante a inalação (respostas que proporcionam dificuldades, atrasos ou impedimentos à execução do procedimento médico) e o aumento na freqüência de emissão de comportamentos de adesão durante a inalação (respostas que não dificultam, antes tendem a facilitar a execução do procedimento médico).

\section{METODOLOGIA}

\section{Participantes}

Os critérios de seleção dos participantes da pesquisa incluíram: 1 ) ter idade entre 5 anos completos e 8 anos e 11 meses; 2) estar internado no setor de enfermaria pediátrica do hospital; 3) apresentar diagnóstico clínico de doença respiratória; 4) ter de submeter-se ao procedimento médico de inalação; e 5) não ser identificado, por meio do Walker check list - WPBIC, como apresentando problemas comportamentais.

Para a pesquisa, procurou-se direcionar a intervenção psicológica a crianças na faixa etária entre 5 e 8 anos de idade, em razão de que teriam um repertório comportamental condizente com a participação e compreensão de atividades previstas no programa.

A seleção de crianças com diagnóstico clínico de doença respiratória (bronquite, pneumonia, broncopneumonia) deveu-se ao fato de que o hospital estava estruturado e atendia com maior freqüência pacientes com tal diagnóstico. O tratamento clínico para tal diagnóstico incluía a realização de inalações, geralmente programadas para cada período de oito horas.

A literatura tem evidenciado que crianças com doenças respiratórias podem apresentar uma maior predisposição para problemas comportamentais, incluindo a baixa auto-estima, transtornos de ansiedade, depressão, pouca competência social e diminuição do rendimento acadêmico (Bennett, 1994; Thompson \& Gustafson, 1996).

Creer \& Bender (1995) descreveram que tal diagnóstico é considerado um dos mais comuns na infância e inclui três aspectos fundamentais: limitação do fluxo aéreo, reversibilidade espontânea ou terapêutica (completa ou parcial) da condição e hiper-atividade brônquica diante de diversos estímulos. O quadro clínico pode envolver dispnéia, sibilos e tosse decorrentes do estreitamento dos condutos aéreos de pequeno calibre e que variam em termos de gravidade. 
Para os autores, fatores comportamentais em interação com mecanismos fisiológicos teriam impacto sobre o curso da doença.

\section{Local}

O presente trabalho foi realizado na enfermaria pediátrica de um hospital público da periferia da cidade de Londrina-PR.

\section{Recursos Materiais e Instrumentos}

Equipamentos: uma câmara de vídeo marca JVC (modelo GR - AXM300U); um videocassete; um cronômetro eletrônico digital marca Technos (modelo TH25).

Material de consumo: Seis fitas de vídeo VHS-C, lápis de cor, canetinhas hidrocor, lápis, papel sulfite, livro de história desenvolvido para a pesquisa, livros infantis (com conteúdos relacionados à doença, à expressão de sentimentos e a intervenções no contexto da saúde), brinquedos e miniaturas de materiais hospitalares.

\section{Instrumentos}

Como instrumentos de coleta de dados, utilizou-se a Escala de Categorias Comportamentais-OSDB e o Walker check list-WPBIC.

Conforme apontaram Peterson, Crowson e Holdridge (1999), a escala OSDB é a escala comportamental mais utilizada para investigar o comportamento de crianças expostas a procedimentos médicos, considerando as diferentes áreas de especialização da Medicina Pediátrica, e tem-se mostrado sensível na identificação de mudanças de comportamento em crianças submetidas a diferentes procedimentos invasivos (Blount, Sturges \& Powers, 1990; Dahlquist, Power, \& Carlson, 1995).

Utilizou-se a redefinição de tal instrumento para a organização de informações obtidas por meio da observação de crianças durante a inalação, incluindo categorias comportamentais definidas como comportamentos concorrentes (choramingar, chorar, comportar-se de modo nervoso, agredir fisicamente, gritar, movimentar-se até a imobilização, fugir e protestar) e comportamentos de adesão (falar, responder verbalmente, solicitar informação, buscar suporte emocional, olhar, olhar para outro estímulo e auxiliar na execução do procedimento médico).

O Walker check list é um instrumento elaborado com base na escala WPBIC (Walker problem behavior identification check list) e utilizado na identificação de crianças com problemas comportamentais, que devem ser encaminhadas para avaliação e posterior atendimento psicológico. Assim, a utilização do WPBIC foi necessária para que, por intermédio de um instrumento confiável, pudessem ser descartados da pesquisa os sujeitos que apresentassem um repertório comportamental deficitário.

\section{Procedimento}

As crianças foram aleatoriamente distribuídas em dois grupos: Grupo experimental (GE), que entrou em contato com as etapas do Programa de Atividades que incluíram a leitura, a simulação, o relaxamento e a fantasia como recursos direcionados à preparação do procedimento médico de inalação; e Grupo-controle (GC), que não entrou em contato com qualquer etapa do Programa de Atividades.

Todas as crianças pertencentes ao GC (10 crianças) e ao GE (10 crianças) foram observadas durante o procedimento médico de inalação nas fases de pré e pós-teste e avaliadas segundo a escala OSDB. Somente crianças identificadas, por meio do WPBIC, como nãoclínicas (sem problemas comportamentais), foram selecionadas para compor a amostra de sujeitos da pesquisa.

A $1^{\text {a }}$ etapa do Programa de Atividades estruturado para crianças do grupo experimental (GE) foi intitulada "Livro infantil" e teve como objetivo: descrever o ambiente hospitalar, informar a função dos profissionais da saúde e a razão dos procedimentos médicos, identificar a percepção da criança com relação à doença e hospitalização, incentivar a verbalização de sentimentos e 
pensamentos em face da condição de hospitalização e de procedimentos médicos.

Para isso, atividades foram estruturadas obedecendo à seguinte seqüência: 1. Leitura de livro infantil elaborado pela equipe; 2 . Solicitação para que a criança conte e/ou desenhe a estória do seu jeito ou conte e/ou desenhe a sua própria estória; 3. Explicitação de questões relacionadas à expressão de sentimentos; 4. Leitura de livros sobre temas relacionados à saúde, à doença, ao medo, à raiva e à expressão de sentimentos; 5 . Disponibilização de figura com desenho do corpo humano.

A $2^{\mathrm{a}}$ etapa do Programa de Atividades foi intitulada de "Brincar de médico" e teve como objetivo: a descrição do procedimento de inalação e outras intervenções médicas (tomar injeção, auscultar, medir temperatura, observar garganta com espátula, fazer curativo e punção); a verbalização de sentimentos em relação a procedimentos médicos, principalmente à inalação; o treinamento de "comportamentos de adesão", aumentando a probabilidade de ocorrência destes comportamentos durante a inalação e a intensificação da relação entre profissional e paciente.

Para alcançar tais objetivos foram disponibilizados brinquedos e materiais utilizados no hospital durante os procedimentos médicos. $O$ desenvolvimento desta etapa consistiu no convite à criança para "brincar de médico", além do fornecimento de informações sobre os procedimentos utilizados, principalmente a inalação.

Para a $3^{\text {a }}$ etapa, intitulada "Relaxamento e Fantasia", foram programadas atividades com o objetivo de aliviar a tensão muscular; produzir imagens relaxantes; analisar funcionalmente o comportamento; identificar variáveis controladoras e formas de intervenção mais eficazes.

Para isso, foram feitas adaptações da técnica de "Relaxamentos para fazer na escola", desenvolvida por Tricoli (2000) e "Relaxamento com imagens", baseado em Markway, Carmin, Pollard e Flynn (1999).

Nessa etapa, buscou-se analisar funcionalmente o comportamento da criança e de outras pessoas, identificando variáveis controladoras e formas de intervenção mais eficazes. A criança também foi solicitada a apontar suas dificuldades diante da aplicação da técnica, sugerindo alternativas para uma intervenção mais apropriada.

\section{Resultados e Discussão}

Os resultados da pesquisa foram analisados estatisticamente e descritivamente. Considera-se que as informações coletadas sobre a criança permitiram a descrição e operacionalização do repertório comportamental dos participantes, o que constitui parte importante da avaliação e intervenção comportamental proposta pela pesquisa.

A análise estatística considerou quão diferentes foram os resultados apresentados pelos grupos nas avaliações conduzidas após o tratamento (Programa de Atividades), comparados aos escores obtidos na linha de base, utilizando a prova dos Sinais e a prova $U$ de Mann-Whitney.

O grupo experimental (GE) e o grupocontrole (GC) foram comparados quanto à freqüência de apresentação das categorias comportamentais pesquisadas, no momento da internação (linha de base) e depois da participação nas três etapas do Programa de Atividades (GE) ou após três períodos diários consecutivos sem participação em qualquer etapa do Programa de Atividades (GC).

Constatou-se, pela análise estatística, que todas as categorias de comportamento do GC não foram estatisticamente diferentes nas fases de pré e pós-teste. No GE, verificou-se diferença significativa entre a fase de pré e de pós-teste, com relação ao "choramingar", "comportar-se de modo nervoso", "protestar" e no total de comportamentos concorrentes. Tais dados indicam que após o tratamento, os sujeitos do GE tiveram uma melhora significativa (diminuição no índice de ocorrência) em comparação aos do GC, nestas categorias.

Quanto à freqüência de emissão de respostas relacionadas aos comportamentos de adesão, dos sujeitos pertencentes ao GE, houve uma sensível diferença na emissão de respostas 
de categorias relacionadas ao "falar", "solicitar informação", "olhar para outro estímulo", "auxiliar na execução do procedimento médico" e somatória total, na situação antes e após o tratamento. O GE, após tratado, demonstrou uma maior freqüência de emissão em comparação ao grupo-controle nessas categorias, demonstrando a eficácia das atividades desenvolvidas no sentido de ampliar um repertório de comportamentos mais adaptativos à situação de inalação.

A análise estatística dos dados por meio da prova U de Mann-Whitney, quando se compararam os comportamentos concorrentes no prée no pós-teste, de sujeitos pertencentes ao grupocontrole e ao grupo experimental, demonstrou diferenças significativas $(p<0,05)$ em relação aos comportamentos D1 (choramingar), D8 (protestar) e TD9 (total). Os demais comportamentos não apresentaram diferenças significativas entre os grupos.

Quando se compararam os comportamentos de adesão, no pré e no pós-teste, de sujeitos pertencentes ao grupo-controle e ao grupo experimental, a análise estatística dos dados, por meio da Prova $U$ de Mann-Whitney, demonstrou diferença significativa $(p<0,05)$ em relação aos comportamentos D9 (falar), D10 (responder verbalmente), D11 (solicitar informação), D12 (buscar suporte emocional) e TD16 (total). Nos demais comportamentos não houve diferença significativa entre os grupos. Relativamente aos comportamentos estatisticamente significativos, o GE apresentou melhora (aumento no índice de ocorrência) quando comparado ao GC.

Pôde-se constatar uma diminuição na ocorrência de categorias de comportamentos concorrentes "choramingar", "protestar" e a somatória total demonstrou ser estatisticamente significativa tanto na prova dos Sinais como na prova U de Mann-Whitney.

Com relação aos comportamentos de adesão, houve aumento na freqüência de ocorrência das categorias "falar", "solicitar informação" e a somatória total demonstrou ser estatisticamente significativa nas duas provas estatísticas acima citadas.
O procedimento utilizado foi mais efetivo para produzir aumento na freqüência de comportamentos de adesão do que para produzir a diminuição na freqüência de comportamentos concorrentes. Tal condição pode ser atribuída ao fato de que, na análise do comportamento (e no procedimento em questão), ficou evidente o incentivo à emissão de comportamentos adaptativos (modelo psicológico de intervenção), já presentes no repertório comportamental do indivíduo, além da instalação de outros. Ao se evidenciar a emissão de comportamentos adaptativos, há uma tendência de aumento na ocorrência dessas habilidades e, naturalmente, uma diminuição na ocorrência de comportamentos não-adaptativos.

A Figura 1 indica o total de ocorrências de comportamentos concorrentes de sujeitos do grupo-controle durante a execução da inalação na fase de pré-teste e de pós-teste.

Na Figura 1 pode-se constatar que a maioria dos sujeitos pertencentes ao grupo-controle apresentaram semelhança no padrão de emissão de comportamentos concorrentes durante a inalação no pré-teste e no pós-teste.

A Figura 2 demonstra o total de ocorrências de comportamentos concorrentes de sujeitos do grupo experimental durante a inalação no préteste e no pós-teste.

A Figura 2 indica que a maioria dos sujeitos pertencentes ao grupo experimental apresentou diminuição considerável na ocorrência de comportamentos concorrentes após terem sido submetidos ao Programa de Atividades.

A Figura 3 demonstra o total de ocorrências de comportamentos de adesão dos sujeitos pertencentes ao grupo-controle durante a inalação na fase do pré-teste (TA) e de pós-teste (TD).

Analisando a Figura 3, pode-se verificar que cinco dos sujeitos pertencentes ao grupo-controle (sujeitos 1, 6, 7, 8 e 10) apresentaram a mesma freqüência de comportamentos de adesão no pré-teste e no pós-teste. O restante dos sujeitos, mesmo sem terem sido submetidos ao Programa de Atividades, tiveram um pequeno aumento na ocorrência de comportamentos de adesão no pós-teste. 


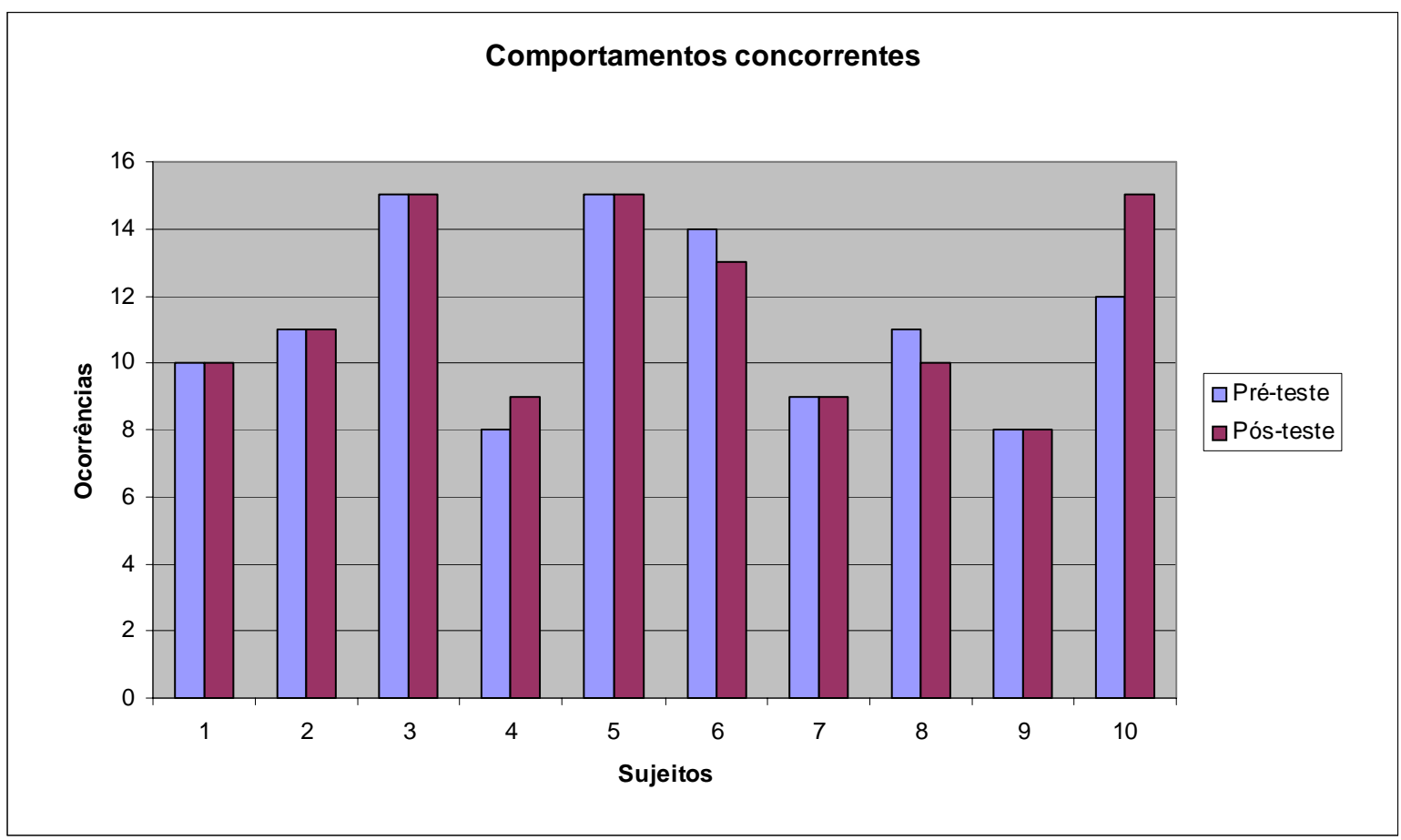

Figura 1. Total de ocorrências de comportamentos concorrentes de sujeitos do grupo-controle durante a inalação na fase de pré-teste e de pós-teste.

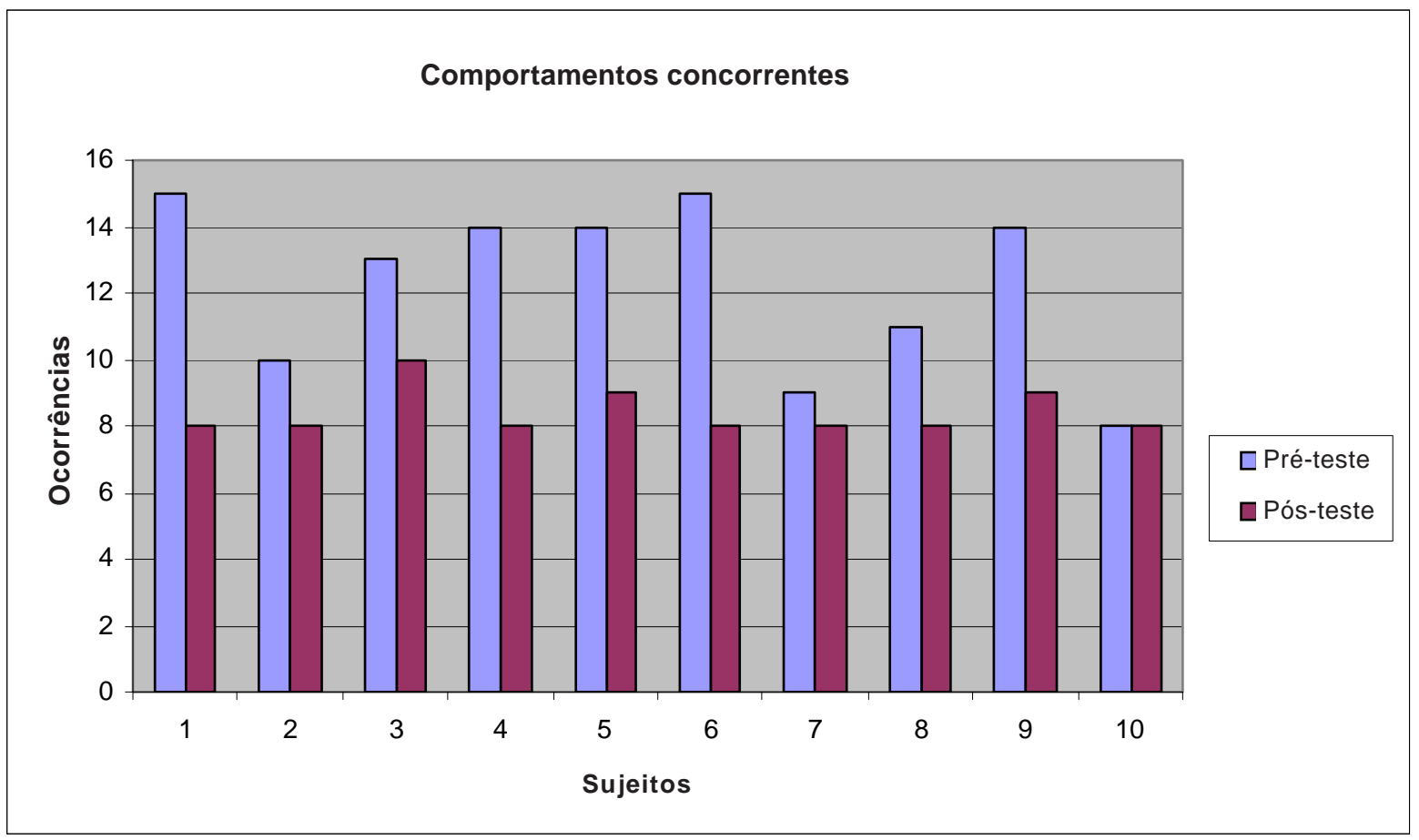

Figura 2. Total de ocorrências de comportamentos concorrentes de sujeitos do grupo experimental durante a inalação na fase de pré-teste e de pós-teste. 


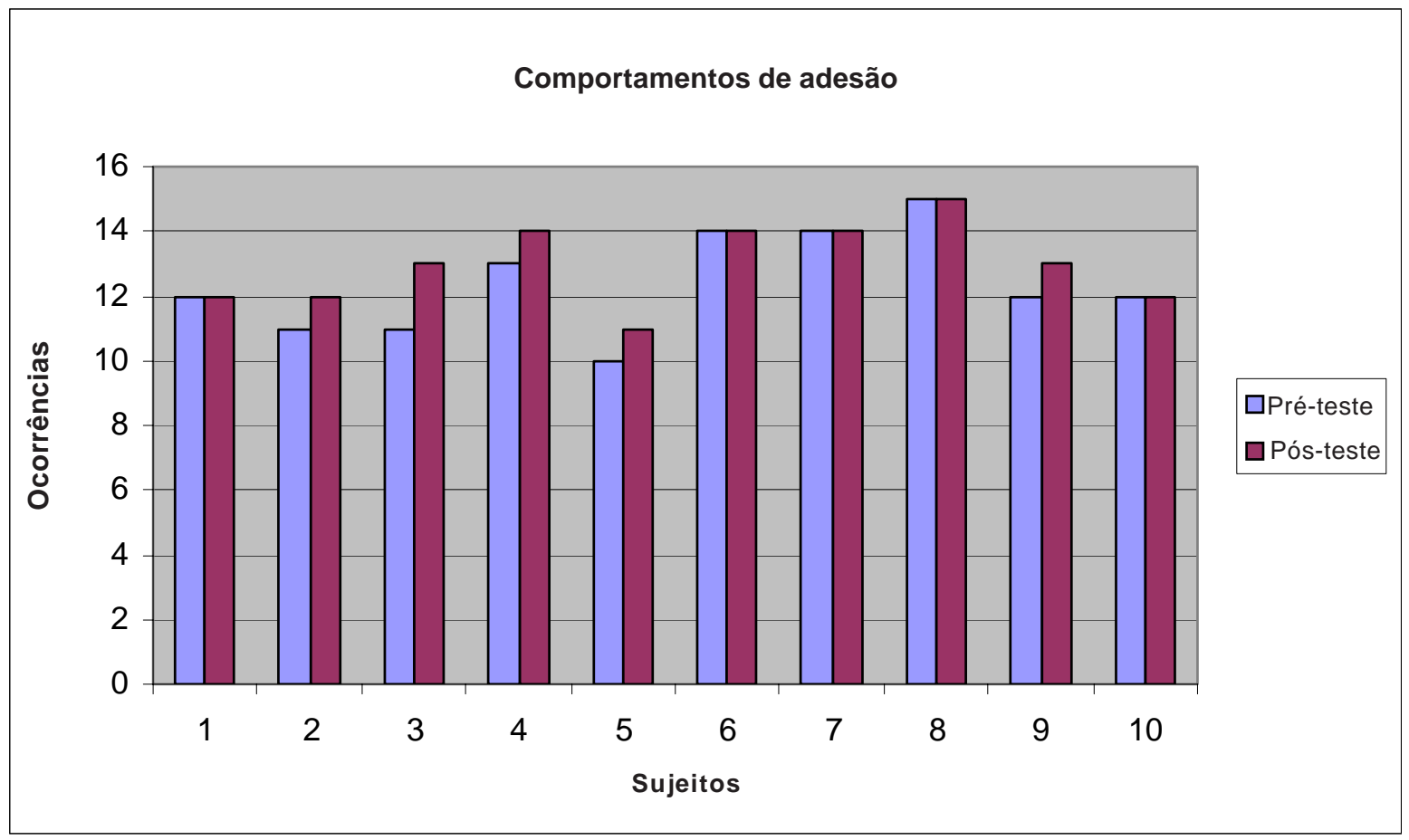

Figura 3. Total de ocorrências de comportamentos de adesão de sujeitos do grupo-controle durante a inalação na fase de pré-teste e de pós-teste.

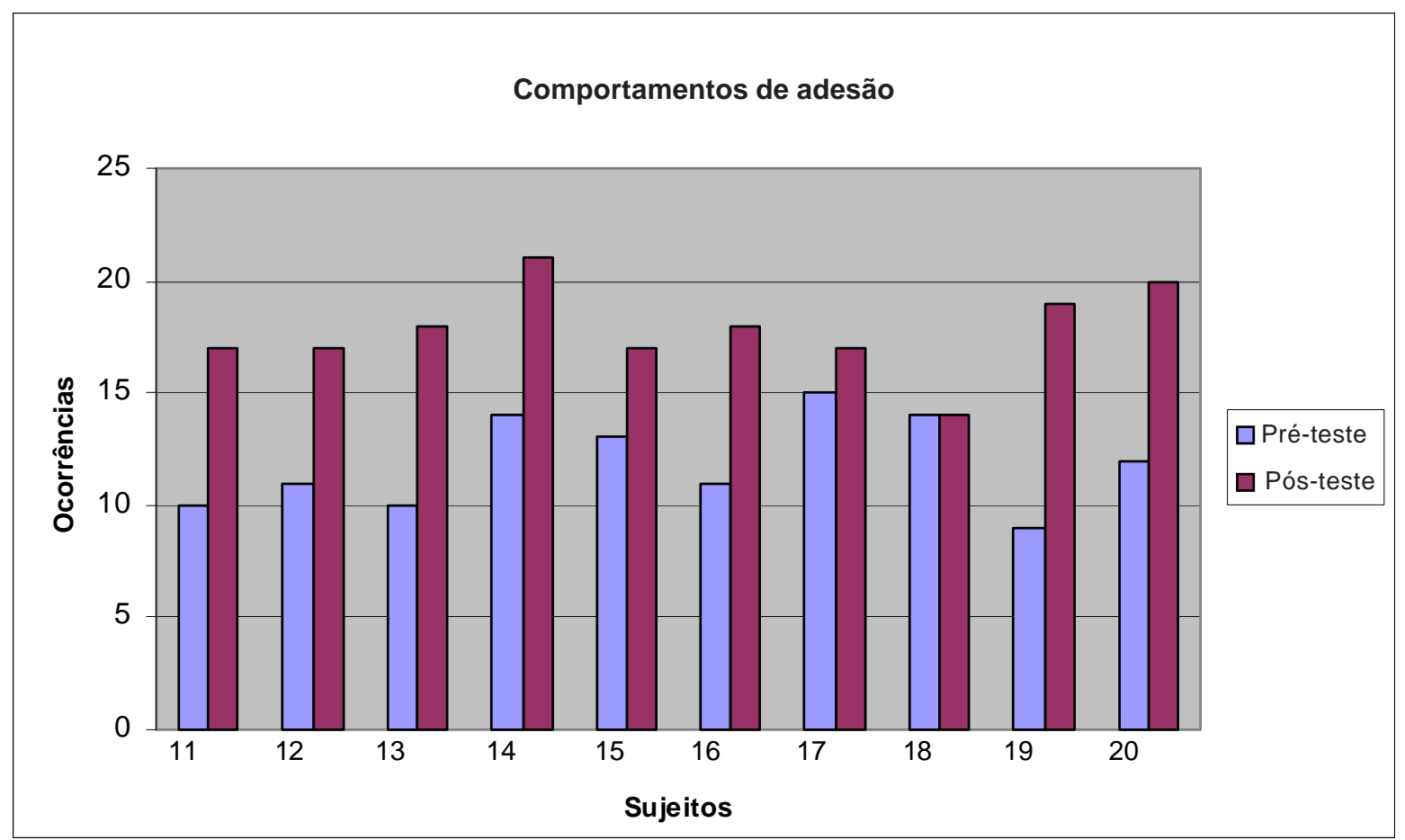

Figura 4. Total de ocorrências de comportamentos de adesão de sujeitos do grupo experimental durante a inalação na fase de pré-teste e de pós-teste. 
Quanto à diminuição de comportamentos concorrentes e ao aumento na freqüência de ocorrência de comportamentos de adesão por parte de sujeitos do grupo-controle, Kole-Kole-Snijders, Goossens, Heuts, Vlaeyen, Mölken \& Breukelen (1999) explicitaram o fato de que durante a hospitalização isso pode acontecer devido ao aumento na tolerância comportamental de crianças, à medida que se expõem a situações desagradáveis (como a de procedimentos médicos, por exemplo).

A Figura 4 indica a ocorrência total de comportamentos de adesão de sujeitos pertencentes ao grupo experimental durante a inalação na fase de pré-teste (TA) e de pós-teste (TD).

Na Figura 4 pode-se verificar que a maioria dos sujeitos pertencentes ao grupo experimental apresentou um aumento considerável na freqüência de ocorrência de comportamentos de adesão após ter sido se submetida ao Programa de Atividades.

Ao comparar a Figura 3 e a Figura 4, podese verificar que mesmo os sujeitos pertencentes ao grupo-controle tiveram aumento na freqüência de ocorrência de comportamentos de adesão no pós-teste; o aumento na freqüência de ocorrência de comportamentos de adesão de sujeitos do grupo experimental foi significativamente maior, o que comprova a eficácia do procedimento da pesquisa.

Pode-se constatar que o Programa de Atividades utilizado, por meio da intervenção breve, foi efetivo para a maioria dos sujeitos do Grupo Experimental, quanto à diminuição na freqüência de comportamentos concorrentes e ao aumento na freqüência de comportamentos de adesão durante o procedimento médico de inalação.

As categorias podem ter ocorrido com maior freqüência no pós-teste dos participantes pertencentes ao Grupo Experimental, pelo fato de que as estratégias do Programa de Atividades buscaram diminuir a percepção de aversividade relacionada ao procedimento médico, facilitando à criança o enfrentamento de tal condição de uma forma mais participativa (falando mais e auxiliando na execução do procedimento médico).

Os resultados obtidos parecem confirmar as observações de Christiano e Russ (1998), referentes à inclusão de programas de atendimento a crianças hospitalizadas, como uma alternativa eficiente para a redução do caráter aversivo dos procedimentos médicos utilizados no contexto hospitalar.

Considerando o grupo de participantes, pode-se afirmar que o procedimento foi efetivo no sentido de reduzir a freqüência de ocorrência de determinados comportamentos concorrentes (principalmente choramingar, comportar-se de modo nervoso e protestar)e de elevar a freqüência de ocorrência de comportamentos de adesão (principalmente falar, olhar para outro estímulo e auxiliar na execução do procedimento médico), à medida que entraram em contato com maior número de informações sobre a condição da doença, tratamento e hospitalização. Tais resultados são coerentes com as observações de Christophersen (1994), segundo o qual, a inclusão de um programa de intervenção psicológica constitui uma oportunidade para que o paciente adquira conhecimento sobre os procedimentos médicos, aumentando a probabilidade de emissão de comportamentos adaptativos e diminuindo a probabilidade de emissão de comportamentos não-adaptativos.

Pela avaliação desses dados, pode-se perceber que as crianças que haviam participado do programa proposto demonstraram melhora considerável em seu padrão comportamental em comparação com crianças pertencentes ao grupocontrole. É justo afirmar que, comparando sujeitos do grupo experimental com sujeitos do grupocontrole, houve diferença na emissão de comportamentos concorrentes e de adesão durante a inalação, no pré e no pós-teste.

Em virtude da freqüência de respostas emitidas por crianças neste estudo, sugere-se que procedimentos que visem à preparação para a inalação devem estar direcionados ao enfoque de respostas relacionadas aos comportamentos concorrentes de "protestar", "choramingar" e 
"comportar-se de modo nervoso". Além disso, devem-se enfatizar os comportamentos adaptativos já presentes no repertório da criança, os quais neste estudo ficaram evidentes, a saber, "falar", "olhar para outro estímulo" e "auxiliar na execução do procedimento médico", demonstrando que tais comportamentos podem ser efetivos para facilitar a adaptação a esse contexto, melhorando a autoconfiança à medida que ela percebe que tem determinadas habilidades em seu repertório.

Constatou-se, por meio dos dados, que meninas tiveram um maior aproveitamento da intervenção psicológica, ou seja, uma maior diferença na freqüência de emissão de comportamentos concorrentes por ocasião do pré e do pós-teste. Apesar desta constatação, não se identificou entre meninos e meninas diferença no nível de aproveitamento pelo teste $t$ de student como significativa $(p<0,05)$, possivelmente devido ao tamanho reduzido da amostra.

O maior aproveitamento do Programa de Atividades por parte das meninas provavelmente se deve ao fato de que elas apresentaram um maior índice de ocorrência de comportamentos concorrentes no pré-teste. Outro fator pode estar relacionado à pouca participação dos meninos na etapa que incluiu principalmente o "Brincar de médico". Supõe-se que questões culturais tenham exercido influência sobre os meninos, fazendo com que apresentassem maior restrição para aceitar brincar com boneca.

Tais dados demonstram que a diferença no desempenho de meninos e meninas em relação à freqüência de emissão de comportamentos durante procedimentos médicos deve ser melhor explicitada em estudos posteriores.

A literatura tem demonstrado que a idade é uma variável que deve ser considerada ao se trabalhar com programas de atendimento com crianças hospitalizadas, porque tal variável pode determinar o sucesso da intervenção (Aragão \& Azevedo, 2001; Araujo \& Arraes, 2000).

Os dados demonstraram que as crianças "menores" (5 a 8 anos de idade) do grupo experimental, independentemente do sexo, se beneficiaram mais do programa proposto pela pesquisa, conseguindo diminuir sensivelmente a freqüência de comportamentos concorrentes. $O$ índice de freqüência de ocorrência dos comportamentos de adesão de todos os sujeitos foi maior após terem sido submetidos ao Programa de Atividades, mas os sujeitos "menores" demonstraram ter-se beneficiado mais do programa, visto que tiveram aumento sensível no índice de ocorrência de comportamentos de adesão.

A utilização da análise funcional possibilitou a avaliação do comportamento das crianças submetidas à inalação, por meio do estabelecimento da relação com o contexto do procedimento médico. Para melhor compreensão do comportamento da criança, foi necessário explicitar elementos relacionados à condição antecedente, fatores disposicionais, repertório comportamental e eventos conseqüentes presentes na situação.

As condições antecedentes relacionaram-se a fatores presentes no ambiente físico (hospital, enfermaria), social (família, profissionais e outras crianças) e estímulos associados ao procedimento de inalação (material para inalação, som do aparelho e presença de equipamentos médicos). As variáveis de ordem organizacional e institucional foram compreendidas como variáveis presentes na rotina hospitalar e que influenciaram na preparação psicológica para procedimentos médicos.

Fatores disposicionais, na medida do possível, devem estar sob um controle mais sistemático e se relacionam com a história de experiência do indivíduo com a doença, crenças, valores, atitudes, rede de apoio social, familiaridade com o ambiente hospitalar, reações à administração de drogas, expectativas com relação aos resultados do trabalho, nível de conhecimento sobre a doença e o tratamento, habilidade dos profissionais da saúde, preferência da criança por determinado agente executor e relação entre profissional e paciente.

Levantando-se a hipótese de que comportamentos concorrentes ocorrem também em virtude 
de estímulos antecedentes presentes em cada fase do procedimento, a compreensão das relações funcionais entre condições antecedentes e comportamentos exibidos pela criança pode permitir um planejamento de intervenção diante de eventos controladores.

Durante o desenvolvimento do Programa de Atividades, optou-se pela utilização de estratégias que incluíram o reforçamento positivo, a modelagem, a modelação, o ensaio comportamental (simulação), a dessensibilização, o fornecimento de informação, o enfrentamento, o autocontrole, o relaxamento, a imaginação, a distração e as atividades lúdicas.

Mesmo considerando tais dificuldades, espera-se que o trabalho possa contribuir para a superação das dificuldades encontradas no atendimento pediátrico em instituições hospitalares brasileiras, fornecendo um modelo de atendimento direcionado à preparação da criança para procedimentos médicos e para superação de dificuldades relacionadas a esse contexto.

Além disso, espera-se que a pesquisa venha acrescentar dados relevantes para a produção científica da área, no sentido não só de diminuir a insuficiência metodológica relacionada ao fornecimento de informações precisas acerca dos efeitos da intervenção psicológica sobre o repertório comportamental do indivíduo hospitalizado, como também de subsidiar intervenções nesse contexto, auxiliando profissionais que atuam no campo da psicologia da saúde a descrever e analisar comportamentos de crianças expostas a procedimentos médicos.

\section{REFERÊNCIAS BIBLIOGRÁFICAS}

ADAMS-Greenly, M. (1991). Psychosocial assessment and intervention at initial diagnosis. Pediatrician, 18, 3-10.

ARAGÃO, R. M. \& Azevedo, M. R. Z. S. (2001). O Brincar no Hospital: Análise de Estratégias e Recursos Lúdicos utilizados com Crianças. Estudos de Psicologia, 18(3), 33-42.

ARAUJO, T. C. C. F. \& Arraes, E. L. M. (2000). Necessidades e expectativas de atuação do psicólogo em cirurgia e procedimentos invasivos. Estudos de Psicologia, 17(1), 6473.

BEARISON, D. J. \& Mulhern, R. K. (1994). Pediatric Psychology - Psychological perspectives on children with cancer. New York: Oxford University Press.

BENNETT, D. S. (1994). Depression among children with chronic medical problems. A meta analysis. Journal of Pediatric Psychology, 19, 149-170.

BLOUNT, R. L. Sturges, J. W., \& Powers, S. W. (1990). Analysis of child and adult behavioral variations by phase of medical procedure. Behavior Therapy, 21, 33-48.

CARPENTER, P. J. (1991). New method for measuring young children's self-report of fear and pain. Journal of Pain and Symptom Management, 5, 233-240.

CHRISTIANO, B. \& Russ, S. W. (1998). Matching preparatory intervention to coping style: The effects on children's distress in the dental setting. Journal of Pediatric Psychology, 23, 17-27.

CHRISTOPHERSEN, E. R. (1994). Pediatric compliance: A guide for the primary care physician. New York: Plenum Medical Book Company.

COSTA Jr. Á. L. (1999). Psiconcologia e Manejo de Procedimentos Invasivos em Oncologia Pediátrica; Uma Revisão de Literatura. Psicologia: Reflexão e Crítica, 12(1), 107-118.

CREER, T. L. \& Bender, B. J. (1995). Pediatric asthma. In M. Roberts (Ed.), Handbook of Pediatric Psychology. New York: Guilford.

DALHQUIST, L. M., Power, T. G., \& Carlson, L. (1995). Physician and parent behavior during invasive pediatric cancer procedures: Relationships to child behavioral distress. Journal of Pediatric Psychology, 20, 477-490.

GUIMARÃES S. S. (1988). A Hospitalizaçãona Infância. Psicologia: Teoria e Pesquisa. 4(2), 102-112.

KOLE-Snijders, A. M. J., Goossens, M. E. J. B., Heuts, P. H. T. G., Vlaeyen, J. W. S., Mölken, 
M. P. M. H. R. \& Breukelen, G. (1999). Chronic Low-Back Pain: What does Cognitive Coping Skills Training Add to Operant Behavioral Treatment? Results of a Randomized Clinical Trial. Journal of Consulting and Clinical Psychology, 67, 931-944.

LAZARUS, R. S. \& Folkman, S. (1984). Stress, appraisal, and coping. New York: SpringerVerlag.

MARKWAY, B. G., Carmin, C. N., Pollard, C. A. \& Flynn, T. (1999). Morrendo de Vergonha: Um guia para tímidos e ansiosos. São Paulo: Summus.

PETERSON, L., Crowson, L. S. \& Holdridge, S. (1999). Of needles and skinned knees: Children's coping with medical procedures and minor injuries of self and other. Health Psychology, 18, 197-200.

RUSHFORTH, H. (1999). Practitioner Review: Communicating with Hospitalizd Children: Review and Application of Research Pertaining to Children's Understanding of Health and Illness. Journal of Child Psychology and Psychiatry, 5, 683-691.
SOUZA, D. G. (1997). O que é contingência? In R. A. Banaco (Org.), Sobre Comportamento e Cognição. Aspectos teóricos, metodológicos e de formação em Análise do Comportamento e Terapia Cognitivista (pp. 82-87). São Paulo: ARBytes.

THOMPSON, R. J. \& Gustafson, K. E. (1996). Adaptation to chronic childhood illness. Washington, D. C.: American Psychological Association.

TRICOLI, V. A. C. (2000). Relaxamentos para fazer na escola. In M. E. N. Lipp (Org.), Crianças Estressadas: Causas, Sintomas e Soluções (pp. 159). Campinas, SP: Papirus.

ZANNON, C. M. L. (1991). Desenvolvimento Psicológico da Criança: Questões básicas relevantes à intervenção comportamental no ambiente hospitalar. Psicologia: Teoria e Pesquisa, 7(2), 119-136.

Recebido para publicação em 1 de setembro de 2003 e aceito em 28 de outubro de 2003. 